\title{
Quantitative Content of a Colloidal Component in a Quartz Glass Based Slip Determination
}

\author{
Kharitonov DV ${ }^{1,2 *}$, Anashkina $\mathrm{AA}^{1,2}$ and Motornova $\mathrm{MS}^{1,2}$ \\ ${ }^{1}$ JSC «ORPE «Technologiya» named after AG Romashin, Obninsk, Russia \\ ${ }^{2}$ D. Mendeleyev University of Chemical Technology of Russia, Moscow, Russia
}

ISSN: 2576-8840

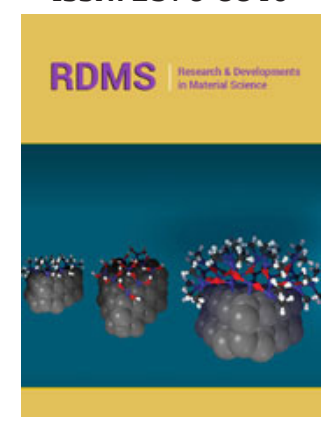

*Corresponding author: Kharitonov DV, JSC «ORPE «Technologiya» named after AG Romashin, Obninsk, Russia

Submission: 侮 May 17, 2019

Published: 㘹 May 24, 2019

Volume 11 - Issue 1

How to cite this article: Kharitonov D, Anashkina A, Motornova M. Quantitative Content of a Colloidal Component in a Quartz Glass Based Slip Determination. Res Dev Material Sci.11(1).RDMS.000752.2019. DOI: 10.31031/RDMS.2019.11.000752

Copyright@ Kharitonov DV, This article is distributed under the terms of the Creative Commons Attribution 4.0 International License, which permits unrestricted use and redistribution provided that the original author and source are credited.

\section{Introduction}

Formation of high-disperse particles of $\mathrm{SiO}_{2}$ in the course of quartz glass-based slip preparation is investigated. The technique of high-disperse silicon dioxide quantitative content in quartz glass-based slip is developed and tested. Concentration of high-disperse particles of $\mathrm{SiO}_{2}$ in various sets of slip is defined. Assessment of colloidal component influence on process of ceramic material sintering is carried out.

Earlier in work [1] the research of formation of high-disperse silicon dioxide particles or a colloidal component in a quartz glass-based slip was carried out. The colloidal component is synthesized in the course of wet quartz glass and represents $\mathrm{SiO}_{2}$ particles from 0 to 340 nanometers in size [1]. Presumably, high-disperse $\mathrm{SiO}_{2}$ particles of act as an activating additive and influence on quartz ceramics sintering process.

To estimate the influence of the colloidal component on properties of ceramic products, it is necessary to know its exact contents in each set of slip. It is necessary to develop a technique of high-disperse $\mathrm{SiO}_{2}$ particles quantitative content in a quartz glass-based slip determination. At the first stage the results of distribution on the sizes of $\mathrm{SiO}_{2}$ particles in each set of slip using laser analyzer of particles size (Figure 1) were analysed.

It is visible that distinctions in percentage of silicon dioxide particles make: for fraction to 0.339 microns - $78 \%$, to 1.000 microns $-65 \%$, to 5.000 microns $-16 \%$. Further the colloidal component was separated from larger fraction of $\mathrm{SiO}_{2}$ particles in a slip using centrifugation. For determination of $\mathrm{SiO}_{2}$ concentration in the allocated dispersion the method was used based on titration of sol by acid in the presence of fluoric sodium. A certain volume of sol was placed in a plastic cup, a small amount of water was added to it and the mixture was titrated by $0,1 \mathrm{~N} \mathrm{HCl}$ in the presence of the methylene red indicator, before disappearance of yellow coloring. Then about $4 \mathrm{~g}$ of $\mathrm{NaF}$ was added. It interacts with c on reaction:

$$
\mathrm{NaOH}+\mathrm{HCl}=\mathrm{NaCl}+\mathrm{H}_{2} \mathrm{O}(1)
$$

The emitted in the course of reaction (1) alkali was titrated when hashing by $1 \mathrm{~N} \mathrm{HCl}$ solution before disappearance of yellow color of the indicator:

$$
\mathrm{NaOH}+\mathrm{HCl}=\mathrm{NaCl}+\mathrm{H}_{2} \mathrm{O}(2)
$$

Titration was finished when the last straw of acid gave to solution steady light pink coloring.

The weight of $\mathrm{SiO}_{2}(\mathrm{~g})$ in $100 \mathrm{~g}$ of dispersion was calculated by a formula:

$$
m_{100 g}\left(\mathrm{SiO}_{2}\right)=1.5 \cdot \frac{\mathrm{V}(\mathrm{HCl}) \cdot \mathrm{C}(\mathrm{HCl})}{m_{\text {sample }}} \text { (3) }
$$

where $\mathrm{V}(\mathrm{HCl})$ - $\mathrm{HCl}$ volume for titration of dispersion, $\mathrm{ml}$; $\mathrm{C}(\mathrm{HCl})$ - concentration of $\mathrm{HCl}, \mathrm{g} / \mathrm{l} ; m_{\text {sample }}$ - mass of a sample of dispersion, $\mathrm{g}$. 

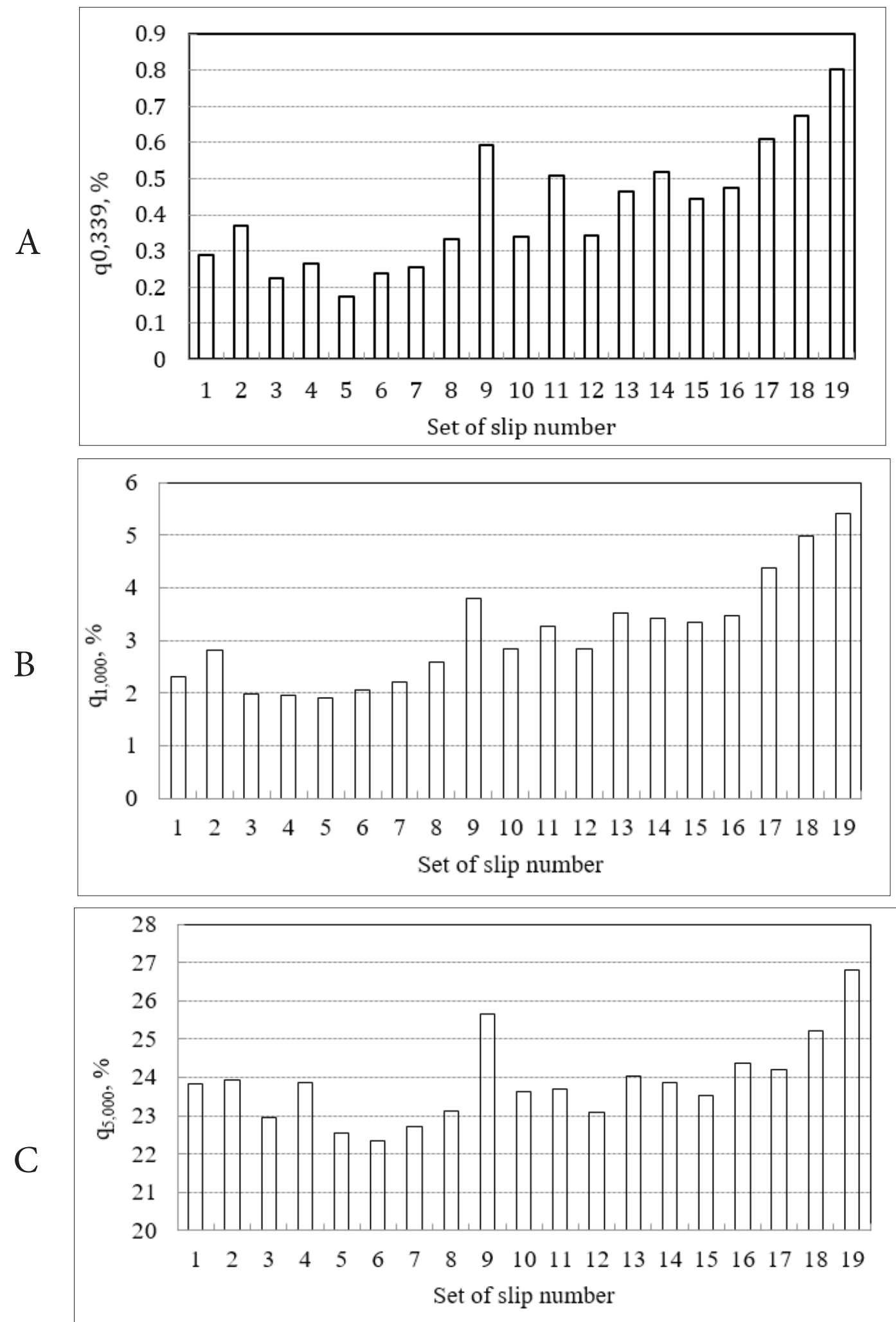

Figure 1: Percentage of particles in slip with sizes to: A-0.339 microns; B-1.000 microns; C-5.000 microns. 
Concentration of the colloidal component in dispersions $(\mathrm{g} / \mathrm{l})$ was calculated by a formulan $\mathrm{C}_{c c}=\frac{\left.\mathrm{SiO}_{2}\right) \cdot \rho_{\text {dispersion }}}{100 \mathrm{~g}}$ (4)

where $\rho_{\text {dispersion }}$ - density of dispersion, g/l.

More exact size is the colloidal component concentration counted for all volume of the slip, or the given colloidal component concentration (\%):

$$
C_{c c}^{g}=\frac{C_{c c} \cdot C_{W}}{C_{V} \cdot \rho_{s}}(5)
$$

\section{Table 1:}

\begin{tabular}{|c|c|c|}
\hline Set of Slip Number & Colloidal Component Concentration $C_{c}, \Gamma / \Omega$ & Given Colloidal Component Concentration $C_{c}^{g}, \%$ \\
\hline 1 & 118.5 & 0.25 \\
\hline 2 & 120.1 & 0.15 \\
\hline 3 & 85.5 & 0.12 \\
\hline 4 & 108.5 & 0.15 \\
\hline 5 & 78.5 & 0.09 \\
\hline 6 & 64.9 & 0.12 \\
\hline 7 & 66.8 & 0.13 \\
\hline 8 & 81.8 & 0.14 \\
\hline 9 & 72.9 & 0.42 \\
\hline 10 & 122.7 & 0.63 \\
\hline 11 & 101.3 & 0.22 \\
\hline 12 & 92.6 & 0.21 \\
\hline 13 & 57.5 & 0.25 \\
\hline 14 & 53.1 & 0.20 \\
\hline 15 & 62.6 & 0.34 \\
\hline 16 & 43.5 & 0.23 \\
\hline 17 & 53.8 & 0.31 \\
\hline 18 & 90.5 & 0.44 \\
\hline 19 & 53.6 & 0.29 \\
\hline
\end{tabular}

\section{Conclusion}

1. The analysis of particles distribution by sizes in each set of slip is carried out.

2. The technique of high-disperse silicon dioxide particles quantitative content determination in a quartz glass-based slip is developed. Values of colloidal component concentration for 19 various sets of slip are calculated.

3. It is shown that the quantitative content of colloidal component in different sets of quartz glass-based slip differs among themselves on several tenth shares of percent. where $\tilde{N}_{W}$-a volume fraction of the disperse medium; $C_{V}$ -volume fraction of the solid phase; $\rho_{s}$-true density of the solid phase, g/l.

\section{Results and Discussion}

Results of definition of colloidal component concentration in dispersion and in slip for various sets of quartz glass-based slip are given in Table 1. It is well seen that for all sets of slip the given colloidal component concentration makes several tenth shares of percent.
4. By results of further researches conclusions on influence of high-disperse silicon dioxide on ceramic material sintering will be drawn.

\section{References}

1. Kharitonov DV, Makarov NA, Anashkina AA, Motornova MS (2018) Effect of highly disperse $\mathrm{SiO}_{2}$ particles on the sintering of quartz ceramic: Firing regime choice for quartz ceramic articles and the colloidal component concept. Glass Ceram 75(10): 190-194. 